\title{
Extensive Air Showers with unusual structure
}

\author{
Dmitriy Beznosko $^{1, a}$, Rashid Beisembaev ${ }^{2}$, Kanat Baigarin ${ }^{1}$, Elena Beisembaeva ${ }^{2}$, Oleg Dalkarov ${ }^{2}$, Vladimir Ryabov $^{2}$, \\ Turlan Sadykov ${ }^{3}$, Sergei Shaulov ${ }^{2}$, Aleksei Stepanov ${ }^{2}$, Marina Vildanova ${ }^{2}$, Nikolay Vildanov ${ }^{2}$, and Valeriy Zhukov ${ }^{2}$ \\ 1 Nazarbayev University, Physics Department, Astana, Kazakhstan \\ 2 P. N. Lebedev Physical Institute of the Russian Academy of Sciences, Moscow, Russia \\ 3 LLP "Institute of Physics and Technology", Almaty, Kazakhstan
}

\begin{abstract}
A total of 23500 Extensive Air Showers (EAS) with energies above $\sim 10^{16} \mathrm{eV}$ have been detected during the $\sim 3500$ hours of the Horizon-T (HT) detectors system operations before Aug. 2016. Among these EAS, more than a thousand had an unusual spatial and temporary structure that showed pulses with several maxima (modals or modes) from several detection points of the HT at the same time. These modes are separated in time from each other starting from tens to thousands of ns. These EAS have been called multimodal. Analysis shows that the multi-modal EAS that have been detected by Horizon-T have the following properties:
\end{abstract}

1. Multi-modal EAS have energy above $\sim 10^{17} \mathrm{eV}$.

2. Pulses with several modes are located at large distances from the EAS axis.

An overview of the collected data will be provided. General comments about the unusual structure of the multi-modal EAS will be presented.

\section{Introduction}

The Horizon-T [1] detector system is located at the Tien Shan High-altitude Science Station (TSHSS) of the Lebedev Physical Institute of the Russian Academy of Sciences at $\sim 3340$ meters above sea level. It consists of eight charged particle detection points, separated by distances up to one kilometer, as well as an optical detector subsystem to measure the Vavilov-Cherenkov light from the EAS. An aerial view of the detector system is shown in Fig. 1. The coordinates of each detection point with respect to the point 1 are given in Table 1 .

HT is an innovative detector system constructed to study both the spatial and temporal distribution of the charged particles within the EAS in the energy range above $10^{16} \mathrm{eV}$ coming from a wide range of zenith angles $\left(0^{\circ}-85^{\circ}\right)$ with a time resolution of a few ns. This novel approach allows to collect two-fold the data from each detection point (particle density and time of passage) and increase the measurement precision of the EAS parameters such as measuring the core position when it falls outside of the detector system area. The HT detector system consists of detectors of several different types: plastic scintillator [2] with MELTS [3] FEU49 model PMT, plastic scintillator with Hamamatsu [4] R7723 PMT assembly, glass with R7723 PMTs and a Vavilov-Cherenkov radiation detector with both FEU49 and Hamamatsu H6527 PMT assemblies. All detection points connect to Data Acquisition (DAQ) via cables that are being calibrated separately [5].

During more than $\sim 3500$ hours of operation from the start of the first prototype up to August 2016 the HT

a e-mail: dmitriy.beznosko@nu.edu. kz detector system detected 23500 EAS with energy above $\sim 10^{16} \mathrm{eV}-$ the threshold energy for HT. Among these EAS, more than a thousand had an unusual spatial and temporal structure that showed pulses with several maxima (also called modes) from several detection points of the HT at the same time. These modes were separated from each other by times from tens to thousands of ns. These EAS are called so called Multi-Modal events (MME). Initially attributed to delayed particles, they were first observed by J. Jelly and W. Whitehouse [6].

\section{Standard EAS definition}

As a solid starting point, the definition of the 'standard' EAS is required. For that, we adopt the output of the EAS simulation package CORSIKA [7]. The simulated EAS serves as a reference that is simple to compare to, and allows to demonstrate certain simple properties of the EAS disk at the observation level.

Firstly, every parent particle regardless of species and energy gives rise to a single uniform EAS without any breaks in the structure or large-scale fluctuations with statistical significance.

Secondly, the particle density drops smoothly with distance when moving away from the core. Symmetry is assumed here for a vertically incoming EAS disk. The charged particles distribution vs. the distance from the core is illustrated in Fig. 2. These simulation results are supported by the result of the experiment 'Hadron' published earlier [8].

Thirdly and finally, the thickness of the EAS disk increases with the distance from the core. Additional characteristics may be sought from the simulated EAS but

(C) The Authors, published by EDP Sciences. This is an Open Access article distributed under the terms of the Creative Commons Attribution License 4.0 (http://creativecommons.org/licenses/by/4.0/). 


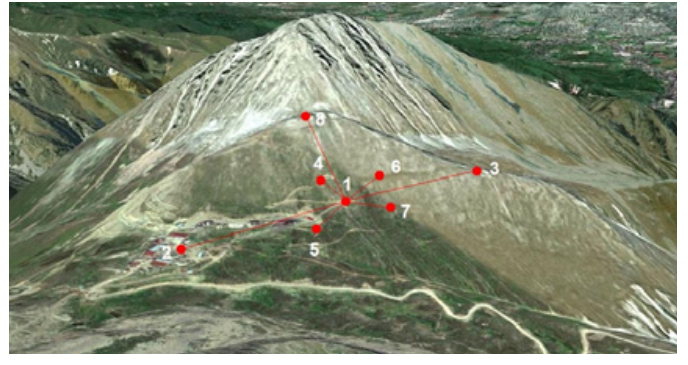

Figure 1. Aerial view of the Horizon-T detector system.

Table 1. HT detection points coordinates.

\begin{tabular}{lrrrr}
\hline Station \# & $\mathrm{X}, \mathrm{m}$ & $\mathrm{Y}, \mathrm{m}$ & $\mathrm{Z}, \mathrm{m}$ & $\mathrm{R}, \mathrm{m}$ \\
\hline 1 & 0 & 0 & 0 & 0 \\
2 & -445.9 & -85.6 & 2.8 & 454.1 \\
3 & 384.9 & 79.5 & 36.1 & 394.7 \\
4 & -55.0 & -94.0 & 31.1 & 113.3 \\
5 & -142.4 & 36.9 & -12.6 & 147.6 \\
6 & 151.2 & -17.9 & 31.3 & 155.4 \\
7 & 88.6 & 178.4 & -39.0 & 203 \\
8 & 221.3 & 262 & 160.7 & 374 \\
\hline
\end{tabular}

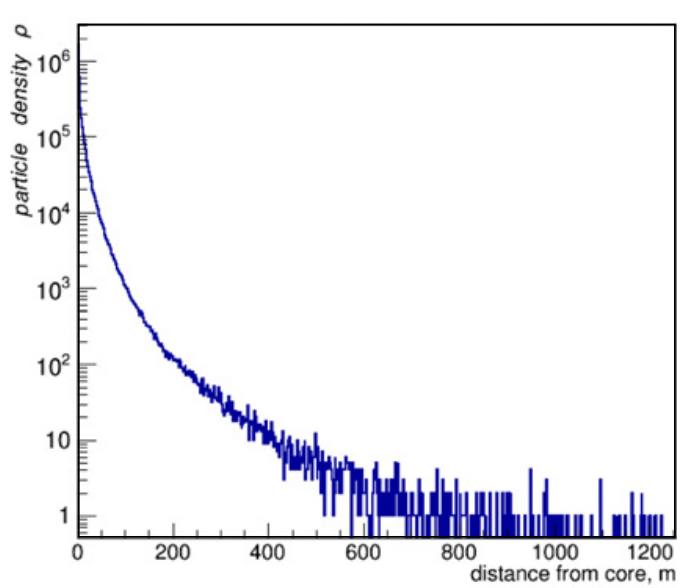

Figure 2. Charged particles density vs. distance from EAS core.

these three seem to be vital ones to both standard definition and the illustration of the unusual structure of the detected EAS. To illustrate the disk widening, the particles arrival time distribution over the $4 \mathrm{~m}^{2}$ area at 50 and 100 meters is shown in Figs. 3 and 4 respectively.

The event display of the standard EAS as detected by the HT detector system is shown in Fig. 5. We can see that the EAS disk arrives from the side of the detector point 4 grazing it with its edge, then hits points 5, 1 and 6 almost simultaneously with the core passing further then hitting point 7 following the arrival direction.

\section{EAS with unusual structure}

Besides the initial observation of the MME in 1953 [6], other scientists were trying to study these events at different times. Initially, the effect was attributed by many of them to the birth in the EAS of a very massive particle with a low $\gamma$ factor that would get delayed and then start a new shower of its own, thus causing the multi-modality of the detected signals.

During the 1960s to 1980s, MME events were studied by British and US physicists, such as Linsley [9],

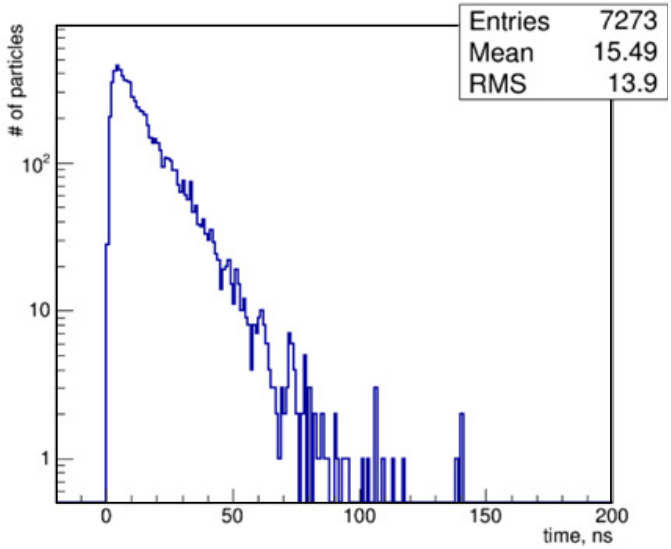

Figure 3. Charged particles arrival time at $50 \mathrm{~m}$ from EAS core.

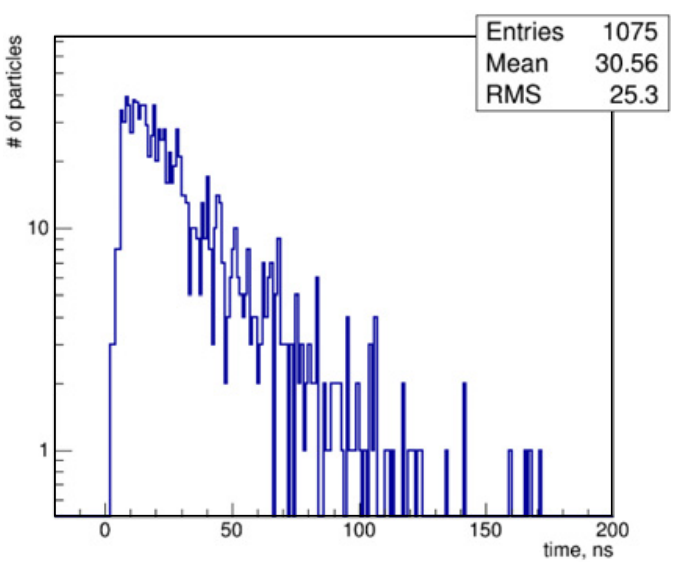

Figure 4. Charged particles arrival time at $100 \mathrm{~m}$ from EAS core.

Watson [10] and Yodh [11]. Since the 1970s, the delayed particle effect has been studied in Japan (Sakuyama [12], Inoue [13]). Starting from the 1980s, studies of MMEs were continued by Moscow State University [14] and at the Lebedev Physical Institute of the Russian Academy of Sciences $[15,16]$. In the 1990s, MME pulses were registered at the Yakutsk detector system [17], and in the 2000's they were detected by the "TUNKA" detector system [18].

Note that the delayed particle explanation, in order to support the time delays observed experimentally, requires frequent birth of particles with rest masses exceeding $10^{12} \mathrm{eV}$. Not only we don't know of such a particle, but, according to current gauge invariance theories, the probability of such a particle being born is inversely proportional to the square of the mass contradicting the frequency of observation. Thus, MME researchers have always admitted that they have extreme difficulty explaining the multi-modality using the current physics knowledge and that MME may indicate the existence of a new physical phenomena.

\subsection{MME example at HT}

Most MME events detected at HT seem to be parts of a larger event arriving far away from the detector system. However, there are several events recorded with the MME axis falling near one of the far periphery points, allowing the observation of the complex structure of such an event. An example of such an MME event is given here. 


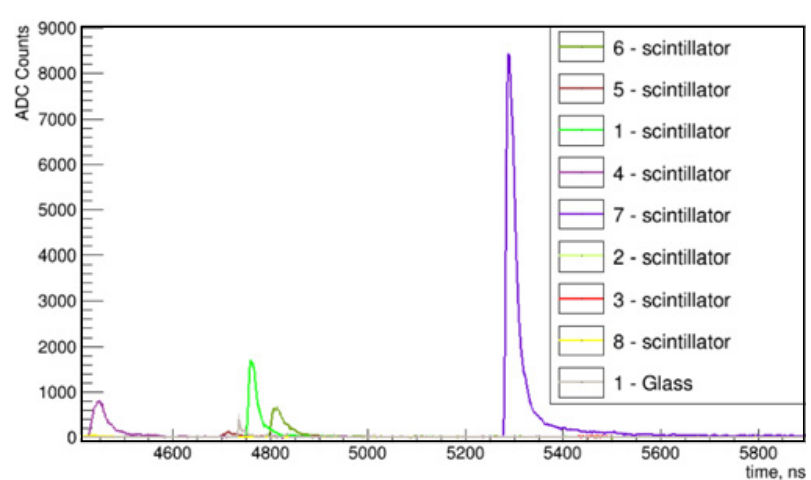

Figure 5. Sample 'standard' event as seen by the HT detector system.

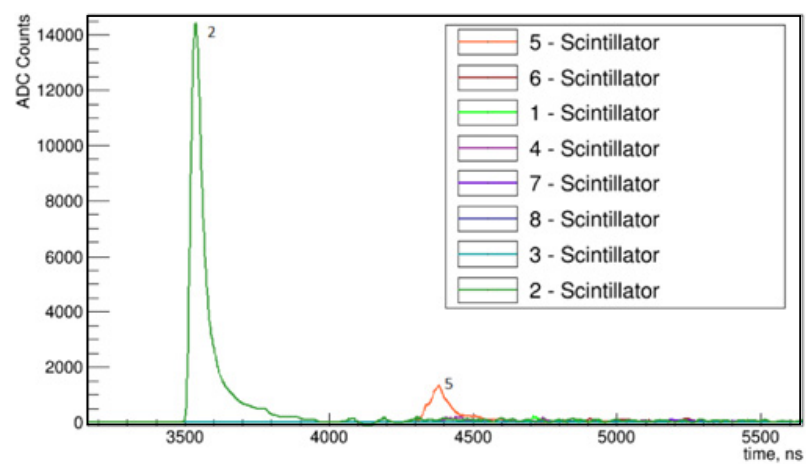

Figure 6. MME as seen by the HT detector system.

The event in Fig. 6 was detected on Jan. 262016 at 3:51 UTC. The event display shows the very large signal at detection point 2 and and the next pulse is visible from point 5, thus indicating that the MME axis has arrived somewhere between these points much closer to point 2 . The amplitudes of pulses from other detection points is much smaller and are not visible in this figure. If we take the pulses with the highest amplitude and use them as for a standard EAS analysis, then the axis of this event is tilted at $\theta \sim 50^{\circ}$ and the arrival direction is approximately along the line, connecting detection points 2 and 3 , and deviating towards point 5 . Note also that there is a pulse from point 2 resembling what is expected from a standard EAS, and the pulse from point 5 seems to start to deviate from that expected of a smooth pulse shape.

Pulses from detection points 1 and 7 only are shown in Fig. 7. Note that these points are both located about the same distance from the axis and further than point 5. Both have a complicated structure, with the signal from point 7 having the modes spread farther apart than for the point 1 signal.

The pulses from detection points 6 and 4 display full multi-modality (see Fig. 8). The peaks are separated by hundreds of ns and feature at least one of the pulses having a complex shape.

The pulses from the detection points 3 and 8 are shown in Fig. 9. These points are furthest from the axis of this event and display the features similar to points 4 and 6 , except that the pulses are further separated by several hundreds of ns. The signal from point 8 has the same 3 distinct modes, however, point 3 has only 2 modes visible. It is also the furthest point from the axis of the event, and it is possible that some modes are too weak to be detected.

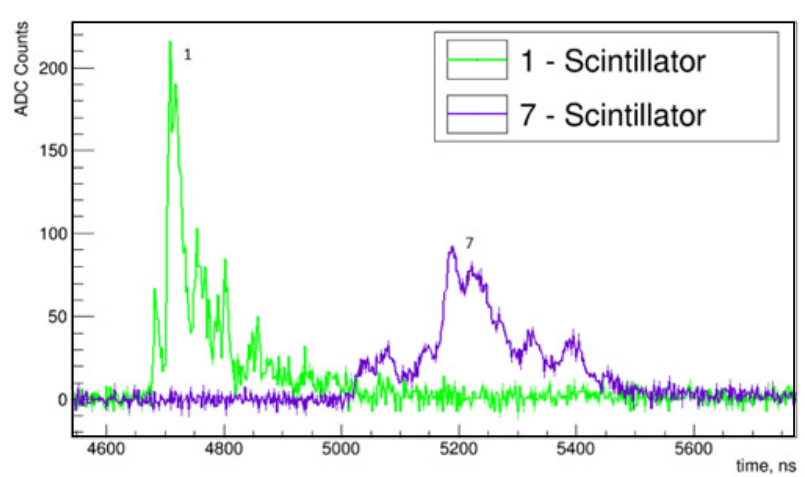

Figure 7. MME as seen by the HT detector system.

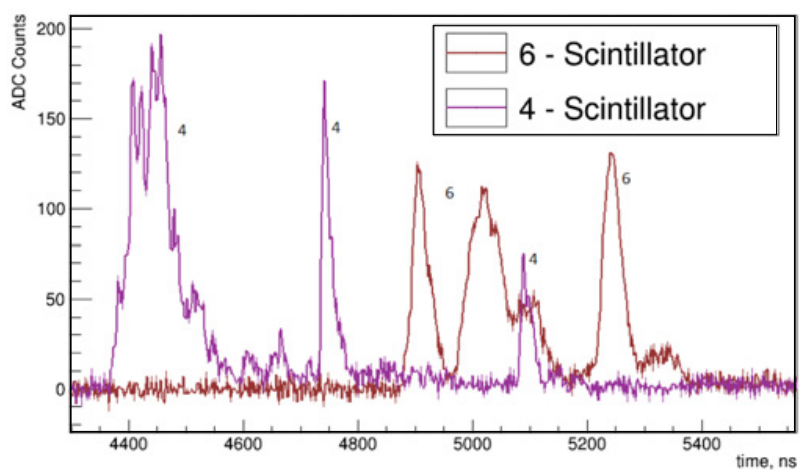

Figure 8. MME as seen by the HT detector system.

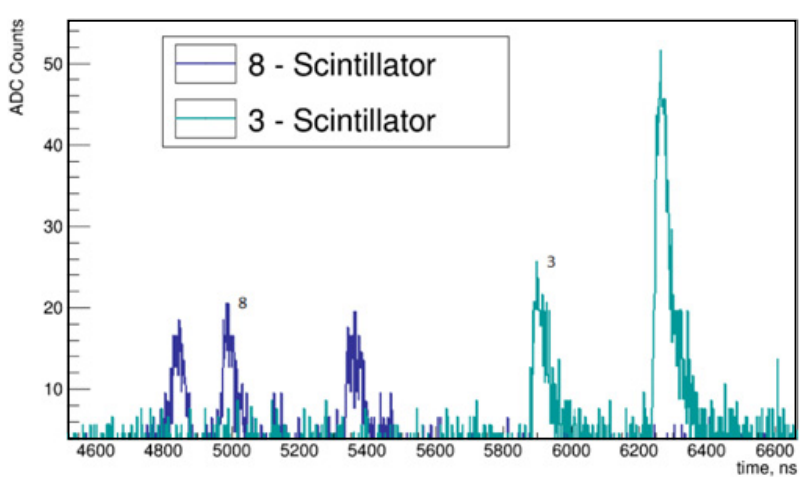

Figure 9. MME as seen by HT detector system.

Using the pulses from all detection points we can infer that this is a very high energy event as its radius is at least $1 \mathrm{~km}$. There are several clean events like this that have been observed at HT and they all follow the same pattern - a large single pulse (that may have structure that is just too fine to be resolved), then the complicated structure pulses at intermediate distances and fully separated multi-peaked pulses at the farthest points from the event axis. It is also plausible that other MME events detected are just parts of the events that have the structure described above. Further studies and analysis of such unusual events are in progress.

\section{Conclusion}

A large number of MME events were detected at HT. The observations show a large variety of shapes and delays between the modes, thus discarding the hypothesis of delayed particles, because in this case all detection points would have shown a similar structure. There is a strong indication that all the variety of forms detected comes from 
the unified structure that was observed in several events with the axis close to the far periphery detection points, allowing observation of the event structure at different distances.

\section{References}

[1] R U Beisembaev et al., arXiv: 1605.05179, (2016)

[2] A Baitenov et al., arXiv:1601.00086, (2016)

[3] MELZ-FEU, 4922-y pr-d, 4c5, Zelenograd, g. Moskva, Russia, 124482 http://www.meltzfeu.ru

[4] Hamamatsu Photonics, 314-5 Shimokanzo, Toyookavillage, Iwatagun, Shizuoka-ken, 438-0193 Japan http: //www.hamamatsu.com

[5] T Beremkulov et al., arXiv: 1608.04312, (2016)

[6] J.V. Jelly and W.J. Whitehouse, Proc. Phys. Soc. A66, 454 (1953)

[7] D. Heck et al., Forschungszentrum Karlsruhe Report FZKA 6019
[8] Adamov D.S. et al., ICRC 460A, 5 (1987)

[9] Linsley J. and Scarsi L., Phys. Rev. 128, 2384 (1962)

[10] Baxter A.J., Watson A.A. and Wilson J.G., Proc. 9 ICRC 2, 724 (1965)

[11] Yodh G.B. et al., Proc. 18 ICRC 11, 264 (1983)

[12] Sakuyama H. et al., Proc. 16 ICRC 8, 135 (1979)

[13] Inoue N. et al., Proc. 19 ICRC 7, 316 (1985)

[14] Atrashevich V. B. et al., RAS News Bul. 58(12), 98 (1994)

[15] Beisembaev R.U. and Vavilov Yu. N. et al., Proc. 24 ICRC. Roma 1, 454 (1995)

[16] Beisembaev R.U. et al., Nuclear Physics T.72. 11, 1-4 (2009)

[17] Glushkov A. B. et al., Letters to Journal of Experimental and Theoretical Physics 67(6), 361 (1998)

[18] Prosin V. V. et al., RAS Bulletin 73(5), 627 (2009) 